\title{
Trace formula for fourth order operators on the circle
}

\author{
Andrey Badanin and Evgeny Korotyaev
}

Communicated by Boris Mityagin, received August 6, 2013.

\begin{abstract}
We determine the trace formula for the fourth order operator on the circle. This formula is similar to the famous trace formula for the Hill operator obtained by Dubrovin, Its-Matveev and McKean-van Moerbeke.
\end{abstract}

\section{Contents}

1. Introduction and main results 343

2. The resolvents 345

3. Proof of Lemma $2.1 \quad 348$

References $\quad 351$

\section{Introduction and main results}

In the present paper we determine the trace formula for the fourth order operator on the circle. Before we recall the famous trace formula for the second order operator $[\mathbf{6}],[\mathbf{7}],[\mathbf{1 1}]$. Consider the differential equation

$$
-y^{\prime \prime}+q y=\lambda y, \quad \lambda \in \mathbb{C},
$$

where $q$ is the 1-periodic potential and $\mathbb{C}$ is a complex plane. Denote by $\alpha_{0}^{+}<$ $\alpha_{1}^{-} \leqslant \alpha_{1}^{+}<\alpha_{2}^{-} \leqslant \ldots$ eigenvalues of the equation (1.1) with the 2-periodic boundary conditions.

Consider the equation (1.1) with the Dirichlet boundary conditions for the shifted potential $q(\cdot+t)$ by any fix parameter $t \in \mathbb{T}=\mathbb{R} / \mathbb{Z}$. Denote the corresponding eigenvalues by $\beta_{1}(t)<\beta_{2}(t)<\ldots$. It is well-known that

$$
\beta_{n}(t) \in\left[\alpha_{n}^{-}, \alpha_{n}^{+}\right] \quad \forall(n, t) \in \mathbb{N} \times \mathbb{T} .
$$

2000 Mathematics Subject Classification. 37, 35.

Key words and phrases. Trace formula, fourth order operator, Hill operator.

(C)2013 International Press 
Now we can recall the famous trace formula, i.e., the identity given by

$$
q(t)=\alpha_{0}^{+}+\sum_{n=1}^{\infty}\left(\alpha_{n}^{+}+\alpha_{n}^{-}-2 \beta_{n}(t)\right) \quad \forall t \in \mathbb{T} .
$$

Dubrovin [6] and Its - Matveev [7] determined the trace formula (1.3) for the socalled $N$ band potential, when $\beta_{n}(t)=\alpha_{n}^{-}=\alpha_{n}^{+}$for all $n \geqslant N, t \in \mathbb{T}$ and some $N \geqslant 1$. In this case the sum in (1.3) is finite. McKean - van Moerbeke [11] and Trubowitz [23] determined the trace formula (1.3) for sufficiently smooth potential. Korotyaev [8] determined the trace formula (1.3) for the case $q \in L^{2}(\mathbb{T})$.

We discuss the trace formula for the fourth order operator. Introduce the Sobolev space $W_{m}^{s}(A)$, where $A$ is the finite interval or the circle, by

$$
W_{m}^{s}(A)=\left\{f, f^{(m)} \in L^{s}(A)\right\}, \quad s \geqslant 1, \quad m=0,1,2, . .
$$

Our main goal is to extend the trace formula (1.3) for the fourth order equation

$$
y^{\prime \prime \prime \prime}+2\left(p y^{\prime}\right)^{\prime}+q y=\lambda y, \quad \lambda \in \mathbb{C},
$$

where the 1-periodic real functions $p, q$ satisfy the conditions

$$
p \in W_{4}^{1}(\mathbb{T}), \quad q \in W_{2}^{1}(\mathbb{T}) .
$$

Consider the equation (1.5) with the 2-periodic boundary conditions. Let

$$
\lambda_{0}^{+}, \lambda_{n}^{ \pm}, n \in \mathbb{N},
$$

be the corresponding spectrum labeled by $\lambda_{0}^{+} \leqslant \lambda_{1}^{-} \leqslant \lambda_{1}^{+} \leqslant \lambda_{2}^{-} \leqslant \ldots$, counted with multiplicities. Note that the eigenvalues have multiplicity $\leqslant 4$. The following asymptotics hold true:

$$
\lambda_{n}^{ \pm}=(\pi n)^{4}-2 p_{0}(\pi n)^{2}+\frac{p_{0}^{2}-\|p\|^{2}}{2}+q_{0}+o\left(n^{-\frac{3}{2}}\right),
$$

as $n \rightarrow+\infty$, see [1] , where

$$
f_{0}=\int_{0}^{1} f(t) d t, \quad\|f\|^{2}=\int_{0}^{1}|f(t)|^{2} d t .
$$

Consider the equation (1.5) with the shifted coefficients by any fix parameter $t \in \mathbb{R}:$

$$
y^{\prime \prime \prime \prime}+2\left(p(x+t) y^{\prime}\right)^{\prime}+q(x+t) y=\lambda y, \quad \lambda \in \mathbb{C},
$$

with the Dirichlet type boundary conditions

$$
y(0)=y^{\prime \prime}(0)=y(1)=y^{\prime \prime}(1)=0 .
$$

Let $\mu_{n}(t), n \geqslant 1$, be the corresponding spectrum labeled by $\mu_{1}(t) \leqslant \mu_{2}(t) \leqslant \mu_{3}(t) \leqslant$ ..., counted with multiplicities. Note that the eigenvalues have multiplicity $\leqslant 2$. The following asymptotics hold true:

$$
\mu_{n}(t)=(\pi n)^{4}-2 p_{0}(\pi n)^{2}+\frac{p_{0}^{2}-\|p\|^{2}}{2}+q_{0}+\frac{O(1)}{n^{2}}
$$

as $n \rightarrow+\infty$ uniformly on $t \in \mathbb{T}$, see $[\mathbf{2}]$.

Now we present trace formulas, which are similar to the second order case (1.3). 
Theorem 1.1. Let $(p, q) \in W_{4}^{1}(\mathbb{T}) \times W_{2}^{1}(\mathbb{T})$. Then the following identity

$$
q(x)-\frac{p^{\prime \prime}(x)}{2}=\lambda_{0}^{+}+\sum_{n=1}^{\infty}\left(\lambda_{n}^{+}+\lambda_{n}^{-}-2 \mu_{n}(x)\right) \quad \forall \quad x \in \mathbb{T},
$$

holds true, where the series converges absolutely and uniformly on $x \in \mathbb{T}$.

In particular, if $p=$ const, then

$$
q(x)=\lambda_{0}^{+}+\sum_{n=1}^{\infty}\left(\lambda_{n}^{+}+\lambda_{n}^{-}-2 \mu_{n}(x)\right) \quad \forall \quad x \in \mathbb{T},
$$

where the series converges absolutely and uniformly on $x \in \mathbb{T}$.

There are numerous results about the trace formulas for the higher order operators, see McKean [10], Ostensson [16], Nazarov, Stolyarov and Zatitskiy [15], Sadovnichii [20], [21], Sadovnichii and Podol'skii [22] and see references therein. The inverse spectral results for the fourth order operators on a finite interval were obtained by Caudill, Perry and Schueller [5], McLaughlin [12], Papanicolaou and Kravvaritis [19]. The spectral problem for the fourth and higher order operator with periodic coefficients considered by Badanin and Korotyaev [3], [4], Mikhailets and Molyboga [14], Papanicolaou [17], [18], Tkachenko [24].

\section{The resolvents}

Let $\mathcal{B}, \mathcal{B}_{1}, \mathcal{B}_{2}$ be the set of all bounded, trace class and Hilbert-Schmidt class operators on $L^{2}(\mathbb{R})$, respectively. The norms of $\mathcal{B}, \mathcal{B}_{1}, \mathcal{B}_{2}$ are denoted by $\|\cdot\|, \| \cdot$ $\left\|_{1},\right\| \cdot \|_{2}$, respectively.

2.1. The second order operators. We recall the following well known results about the second order operators $h_{1}, h_{2}$, see, for instance, in [13], [9].

Firstly, we consider the operator $h_{2} y=-y^{\prime \prime}-p y$ acting in $L^{2}(0,2)$ with the 2-periodic boundary conditions. The spectrum of $h_{2}$ is discrete and its eigenvalues $\alpha_{0}^{+}, \alpha_{n}^{ \pm}, n \in \mathbb{N}$, are simple or have multiplicity two. They can enumerated, counting with multiplicity, so that $\alpha_{0}^{+}<\alpha_{1}^{-} \leqslant \alpha_{1}^{+}<\alpha_{2}^{-} \leqslant \alpha_{2}^{+}<\ldots$ The following asymptotics hold true:

$$
\alpha_{n}^{ \pm}=(\pi n)^{2}+O(1) \quad \text { as } n \rightarrow+\infty .
$$

Secondly, we consider the operator $h_{1}$ acting in $L^{2}(0,1)$ with the Dirichlet boundary conditions given by

$$
h_{1} y=-y^{\prime \prime}-p y, \quad y(0)=y(1)=0 .
$$

All eigenvalues $\beta_{n}, n \in \mathbb{N}$, of the operator $h_{1}$ are simple. We enumerate their in the increasing order $\beta_{1}<\beta_{2}<\ldots$ Recall that $\beta_{n} \in\left[\alpha_{n}^{-}, \alpha_{n}^{+}\right]$for all $n \geqslant 1$. Moreover, the following trace formula holds true:

$$
p^{2}(0)+\frac{p^{\prime \prime}(0)}{2}=\left(\alpha_{0}^{+}\right)^{2}+\sum_{n \geqslant 1}\left(\left(\alpha_{n}^{+}\right)^{2}+\left(\alpha_{n}^{-}\right)^{2}-2 \beta_{n}^{2}\right)
$$

where the series converges absolutely, see [11, p.254]. 
2.2. The fourth order operators. Introduce the self-adjoint operator $H_{2}$ in $L^{2}(0,2)$ by

$$
H_{2}=\partial^{4}+2 \partial p \partial+q \quad \text { on } \quad[0,2]
$$

with 2-periodic boundary conditions.

It is sufficiently to prove identity $(1.11)$ at $x=0$. Thus we need to define the operator $H_{1}$ in $L^{2}(0,1)$ by

$$
H_{1}=\partial^{4}+2 \partial p \partial+q \text { on }[0,1]
$$

with the Dirichlet type boundary conditions

$$
f(0)=f^{\prime \prime}(0)=f(1)=f^{\prime \prime}(1)=0 .
$$

Using the identity

$$
\partial^{4}+2 \partial p \partial+q=\left(-\partial^{2}-p\right)^{2}+V, \quad V=q-p^{\prime \prime}-p^{2},
$$

and $V \in L^{\infty}(0,1)$, we obtain

$$
H_{j}=h_{j}^{2}+V, \quad j=1,2,
$$

where the operators $h_{1}$ and $h_{2}$ are introduced above. Let the operator $h_{j}^{0}, j=1,2$, be equal to the operator $h_{j}$ at $p=0$. Introduce the resolvents

$$
R_{j}(\lambda)=\left(H_{j}-\lambda\right)^{-1}, \quad \mathcal{R}_{j}(\lambda)=\left(h_{j}^{2}-\lambda\right)^{-1}, \quad \mathcal{R}_{j}^{0}(\lambda)=\left(\left(h_{j}^{0}\right)^{2}-\lambda\right)^{-1},
$$

where $j=1,2$. We have

$$
\mathcal{R}_{j}=\mathcal{R}_{j}^{0}-\mathcal{R}_{j}\left(-h_{j}^{0} p-p h_{j}^{0}+p^{2}\right) \mathcal{R}_{j}^{0} .
$$

Due to asymptotics (1.7), (1.10), (2.1) all resolvents satisfy

$$
\mathcal{R}_{j}(\lambda), R_{j}(\lambda), h_{j}^{0} \mathcal{R}_{j}^{0}(\lambda) \in \mathcal{B}_{1}
$$

on the corresponding resolvent sets. Define the contours $K_{n} \subset \mathbb{C}$ by

$$
K_{n}=\left\{\lambda \in \mathbb{C}:|\lambda|^{\frac{1}{4}}=\pi\left(n+\frac{1}{2}\right)\right\}, \quad n \geqslant 1 .
$$

We present results about the asymptotics, proved in Section 3.

Lemma 2.1. Let $j=1,2$ and let $n \rightarrow \infty$. Then the following asymptotics hold true:

$$
\begin{gathered}
\left\|\mathcal{R}_{j}^{0}(\lambda)\right\|_{2}+\left\|\mathcal{R}_{j}(\lambda)\right\|_{2}+\left\|R_{j}(\lambda)\right\|_{2}=O\left(n^{-3}\right) \\
\left\|h_{j}^{0} \mathcal{R}_{j}^{0}(\lambda)\right\|_{2}=O\left(n^{-1}\right)
\end{gathered}
$$

uniformly on the contours $K_{n}$, and

$$
\oint_{K_{n}}\left(\lambda \operatorname{Tr} V \mathcal{R}_{j}^{2}(\lambda)+\operatorname{Tr} V \mathcal{R}_{j}^{0}(\lambda)\right) d \lambda=o(1) .
$$


2.3. Proof of the main results. Introduce the function

$$
\Phi=\operatorname{Tr}\left(R_{2}-\mathcal{R}_{2}\right)-2 \operatorname{Tr}\left(R_{1}-\mathcal{R}_{1}\right) .
$$

Lemma 2.2. The following identity holds true:

$$
\lim _{n \rightarrow+\infty} \frac{1}{2 \pi i} \oint_{K_{n}} \lambda \Phi(\lambda) d \lambda=-V(0) .
$$

Proof. Substituting the identities

$$
R_{j}=\mathcal{R}_{j}-R_{j} V \mathcal{R}_{j}=\mathcal{R}_{j}-\mathcal{R}_{j} V \mathcal{R}_{j}+R_{j} V \mathcal{R}_{j} V \mathcal{R}_{j}, \quad j=1,2,
$$

into (2.14) we obtain

$$
\Phi=\Phi_{0}+\Phi_{1}
$$

where

(2.17) $\Phi_{0}=-\operatorname{Tr} V \mathcal{R}_{2}^{2}+\operatorname{Tr} 2 V \mathcal{R}_{1}^{2}, \quad \Phi_{1}=\operatorname{Tr} V \mathcal{R}_{2} V \mathcal{R}_{2} R_{2}-\operatorname{Tr} 2 V \mathcal{R}_{1} V \mathcal{R}_{1} R_{1}$

Let $n \rightarrow \infty$. Identities (2.17), (2.13) yield

$$
\oint_{K_{n}}\left(\lambda \Phi_{0}(\lambda)-F(\lambda)\right) d \lambda=o(1)
$$

where

$$
F=\operatorname{Tr} V \mathcal{R}_{2}^{0}-2 \operatorname{Tr} V \mathcal{R}_{1}^{0}
$$

Estimates (2.11) give

$$
\left|\operatorname{Tr} V \mathcal{R}_{j}(\lambda) V \mathcal{R}_{j}(\lambda) R_{j}(\lambda)\right| \leqslant\left\|R_{j}(\lambda)\right\|_{2}\left\|\mathcal{R}_{j}(\lambda)\right\|_{2}^{2}\|V\|^{2}=O\left(n^{-9}\right), \quad j=1,2,
$$
uniformly on contours $K_{n}$, which yields $\Phi_{1}(\lambda)=O\left(n^{-9}\right)$ and then

$$
\oint_{K_{n}} \lambda \Phi_{1}(\lambda) d \lambda=o(1)
$$

Then identity (2.16) gives

$$
\oint_{K_{n}}(\lambda \Phi(\lambda)-F(\lambda)) d \lambda=o(1)
$$

We have the Fourier series

$$
V(x)=V_{0}+2 \sum_{n=1}^{\infty}\left(V_{c n} \cos 2 \pi n x+V_{s n} \sin 2 \pi n x\right),
$$

where

$$
V_{s n}=\int_{0}^{1} V(x) \sin 2 \pi n x d x, \quad V_{c n}=\int_{0}^{1} V(x) \cos 2 \pi n x d x, \quad V_{0}=\int_{0}^{1} V(x) d x .
$$

Let $e_{n}=\frac{1}{\sqrt{2}} e^{i \pi n x}, n \in \mathbb{Z}$, and $s_{n}=\sin \pi n x, n \in \mathbb{N}$. Define the scalar products $\langle f, g\rangle=\int_{0}^{2} f \bar{g} d x$ and $(f, g)=\int_{0}^{1} f \bar{g} d x$ in $L^{2}(0,2)$ and $L^{2}(0,1)$ respectively. Identity (2.18) gives

$$
\begin{aligned}
& F(\lambda)=\frac{\left\langle V e_{0}, e_{0}\right\rangle}{-\lambda}+\sum_{n=1}^{\infty} \frac{\left\langle V e_{n}, e_{n}\right\rangle+\left\langle V e_{-n}, e_{-n}\right\rangle-4\left(V s_{n}, s_{n}\right)}{(\pi n)^{4}-\lambda} \\
& =-\frac{V_{0}}{\lambda}+\sum_{n=1}^{\infty} \frac{2 V_{c n}}{(\pi n)^{4}-\lambda},
\end{aligned}
$$


since we have the identities

$$
\begin{gathered}
\left\langle V e_{n}, e_{n}\right\rangle=V_{0} \quad \forall n \in \mathbb{Z} \\
\left(V s_{n}, s_{n}\right)=\int_{0}^{1} V \sin ^{2} \pi n x d x=\frac{V_{0}-V_{c n}}{2}, \quad n \geqslant 1 .
\end{gathered}
$$

Then

$$
\frac{1}{2 \pi i} \oint_{K_{N}} F(\lambda) d \lambda=-V_{0}-2 \sum_{n=1}^{N} V_{c n}
$$

for all $N \geqslant 1$. Identity (2.19) give

$$
\frac{1}{2 \pi i} \oint_{K_{N}} \lambda \Phi(\lambda) d \lambda=-\left(V_{0}+2 \sum_{n=1}^{N} V_{c n}\right)+o(1) \quad \text { as } \quad N \rightarrow \infty,
$$

then (2.20) yields (2.15).

Proof of Theorem 1.1. Asymptotics (1.7), (1.10) show that the series

$$
S=\lambda_{0}^{+}+\sum_{n \geqslant 1}\left(\lambda_{n}^{+}+\lambda_{n}^{-}-2 \mu_{n}\right)
$$

converges absolutely. Let $S_{0}$ be given by

$$
S_{0}=\left(\alpha_{0}^{+}\right)^{2}+\sum_{n \geqslant 1}\left(\left(\alpha_{n}^{+}\right)^{2}+\left(\alpha_{n}^{-}\right)^{2}-2 \beta_{n}^{2}\right)
$$

Then due to (2.14) we have

$$
S-S_{0}=-\frac{1}{2 \pi i} \lim _{n \rightarrow+\infty} \oint_{K_{n}} \lambda \Phi(\lambda) d \lambda .
$$

Identity (2.3) yields

$$
S-p^{2}(0)-\frac{p^{\prime \prime}(0)}{2}=-\frac{1}{2 \pi i} \lim _{n \rightarrow+\infty} \oint_{K_{n}} \lambda \Phi(\lambda) d \lambda .
$$

Substituting identity (2.15) into (2.21) we obtain

$$
S-p^{2}(0)-\frac{p^{\prime \prime}(0)}{2}=V(0) .
$$

Substituting (2.5) into the last identity we have $S=q(0)-\frac{p^{\prime \prime}(0)}{2}$, which yields (1.11).

\section{Proof of Lemma 2.1}

Proof of Lemma 2.1. We will prove (2.11) for $R_{1}(\lambda)$. The proof for other $R_{2}, \mathcal{R}_{j}, \mathcal{R}_{j}^{0}$ is similar. Let

$$
a_{k}=\left|\mu_{k}\right|^{\frac{1}{4}}, \quad a=|\lambda|^{\frac{1}{4}}, \quad \lambda \in K_{n}, \quad k, n \geqslant 1 .
$$

Then we have

$$
\left|\mu_{k}-\lambda\right| \geqslant|| \mu_{k}|-| \lambda||=\left|a_{k}-a\right|\left(a_{k}+a\right)\left(a_{k}^{2}+a^{2}\right) \geqslant\left|a_{k}-a\right| a^{3} .
$$

Asymptotics (1.10) yields

$$
\begin{gathered}
a_{k}=\pi k+\pi \varepsilon_{k}, \quad \text { where } \quad\left|\varepsilon_{k}\right|<\frac{1}{4} \quad \forall k>N, \\
\left|a_{k}-a\right| \geqslant 1 \quad \forall k \leqslant N
\end{gathered}
$$


for $n \in \mathbb{N}$ large enough and for some $N \in \mathbb{N}$ large enough. We have

$$
\left|a_{k}-a\right|=\pi\left|k-n-\frac{1}{2}+\varepsilon_{k}\right| \geqslant \pi\left(|k-n|-\frac{1}{4}\right) \quad \forall \quad k>N .
$$

Estimates $(3.1)-(3.4)$ give

$$
\begin{aligned}
& \left\|R_{1}(\lambda)\right\|_{2}^{2}=\sum_{k=1}^{\infty} \frac{1}{\left|\mu_{k}-\lambda\right|^{2}} \leqslant \frac{1}{a^{6}} \sum_{k=1}^{\infty} \frac{1}{\left|a_{k}-a\right|^{2}} \\
& \leqslant \frac{1}{a^{6}}\left(N+\sum_{k=N+1}^{\infty} \frac{1}{\pi^{2}\left(|k-n|-\frac{1}{4}\right)^{2}}\right) \leqslant \frac{C}{a^{6}},
\end{aligned}
$$

where $C=N+\frac{1}{\pi^{2}} \sum_{k \in \mathbb{Z}}\left(k-\frac{1}{4}\right)^{-2}<\infty$ and this yields (2.11) for $R_{1}(\lambda)$.

We will prove $(2.12)$ for $j=1$. The proof for $j=2$ is similar. We have

$$
\begin{aligned}
& \left|(\pi k)^{4}-\lambda\right| \geqslant||(\pi k)^{4}-|\lambda||=| \pi k-a \mid(\pi k+a)\left((\pi k)^{2}+a^{2}\right) \\
& \geqslant a|\pi k-a|\left((\pi k)^{2}+a^{2}\right) .
\end{aligned}
$$

This estimate implies

$\left\|h_{j}^{0} \mathcal{R}_{j}^{0}(\lambda)\right\|_{2}^{2}=\sum_{k=1}^{\infty} \frac{(\pi k)^{4}}{\left|(\pi k)^{4}-\lambda\right|^{2}} \leqslant \frac{1}{a^{2}} \sum_{k=1}^{\infty} \frac{1}{|\pi k-a|^{2}} \leqslant \frac{1}{\pi^{2} a^{2}} \sum_{k=1}^{\infty} \frac{1}{\left|k-n-\frac{1}{2}\right|^{2}} \leqslant \frac{C_{1}}{a^{2}}$,

where $C_{1}=\frac{1}{\pi^{2}} \sum_{k \in \mathbb{Z}}\left(k-\frac{1}{2}\right)^{-2}<\infty$, which yields (2.12) for $j=1$.

We will prove asymptotics (2.13) for $j=2$. The proof for $j=1$ is similar. The integration by parts gives

$$
\oint_{K_{n}} \lambda \operatorname{Tr} V \mathcal{R}_{2}^{2}(\lambda) d \lambda=\oint_{K_{n}} \lambda \operatorname{Tr} V\left(\mathcal{R}_{2}(\lambda)\right)^{\prime} d \lambda=-\oint_{K_{n}} \operatorname{Tr} V \mathcal{R}_{2}(\lambda) d \lambda
$$

for all $n \in \mathbb{N}$. Using identity (2.8) we obtain

$$
\begin{aligned}
& \oint_{K_{n}}\left(\lambda \operatorname{Tr} V \mathcal{R}_{2}^{2}(\lambda)+\operatorname{Tr} V \mathcal{R}_{2}^{0}(\lambda)\right) d \lambda=\oint_{K_{n}} \operatorname{Tr} V\left(\mathcal{R}_{2}^{0}(\lambda)-\mathcal{R}_{2}(\lambda)\right) d \lambda \\
& =\oint_{K_{n}} \operatorname{Tr}\left(V \mathcal{R}_{2}(\lambda) Q \mathcal{R}_{2}^{0}(\lambda)\right) d \lambda, \quad \text { where } \quad Q=-h_{2}^{0} p-p h_{2}^{0}+p^{2} .
\end{aligned}
$$

Asymptotics (2.11), (2.12) give

$$
\begin{aligned}
& \left|\operatorname{Tr}\left(\mathcal{R}_{2}^{0}(\lambda) Q(\lambda) \mathcal{R}_{2}^{0}(\lambda) Q(\lambda) \mathcal{R}_{2}(\lambda)\right)\right| \\
& \leqslant\left\|\mathcal{R}_{2}^{0}(\lambda) Q(\lambda)\right\|_{2}^{2}\left\|\mathcal{R}_{2}(\lambda)\right\|_{2}=O\left(n^{-5}\right) \text { as } n \rightarrow \infty
\end{aligned}
$$

uniformly on all contours $K_{n}$. Substituting (2.8) into (3.6) and using (3.7) we obtain

$$
\begin{aligned}
& \oint_{K_{n}}\left(\lambda \operatorname{Tr} V \mathcal{R}_{2}^{2}(\lambda)+\operatorname{Tr} V \mathcal{R}_{2}^{0}(\lambda)\right) d \lambda \\
& =\oint_{K_{n}} \operatorname{Tr} V \mathcal{R}_{2}^{0}(\lambda) Q \mathcal{R}_{2}^{0}(\lambda) d \lambda+o(1) \quad \text { as } \quad n \rightarrow \infty
\end{aligned}
$$


Thus we need to consider $\operatorname{Tr} V \mathcal{R}_{2}^{0}(\lambda) Q \mathcal{R}_{2}^{0}(\lambda)$, where $Q=p^{2}-h_{2}^{0} p-p h_{2}^{0}$. Firstly, we consider the case $p^{2}$. Estimate $(2.11)$ as $n \rightarrow \infty$ yields

$$
\begin{aligned}
& \oint_{K_{n}} \operatorname{Tr} V \mathcal{R}_{2}^{0}(\lambda) p^{2} \mathcal{R}_{2}^{0}(\lambda) d \lambda=O(1) \oint_{K_{n}}\left\|\mathcal{R}_{2}^{0}(\lambda)\right\|_{2}^{2}|d \lambda| \\
& =\oint_{K_{n}} O\left(n^{-6}\right)|d \lambda|=O\left(n^{-2}\right) .
\end{aligned}
$$

Secondly, we consider the case $-h_{2}^{0} p-p h_{2}^{0}$. Using $\widehat{f}_{k}=\int_{0}^{2} f(x) e^{i \pi k x} d x$ and the identity

$\left\langle\left(-h_{2}^{0} p-p h_{2}^{0}\right) e_{m}, e_{k}\right\rangle=\int_{0}^{2} e^{-i \pi k x}\left(\partial_{x}^{2} p(x)+p(x) \partial_{x}^{2}\right) e^{i \pi m x} d x=-\pi^{2}\left(k^{2}+m^{2}\right) \widehat{p}_{m-k}$,

where $e_{m}=\frac{1}{\sqrt{2}} e^{i \pi m x}$ and $\langle f, g\rangle=\int_{0}^{2} f \bar{g} d x$, we have

$$
\begin{aligned}
& \operatorname{Tr}\left(V \mathcal{R}_{2}^{0}\left(h_{2}^{0} p+p h_{2}^{0}\right) \mathcal{R}_{2}^{0}\right)=-\sum_{k=-\infty=-\infty}^{\infty} F(k, m, \lambda) \\
& =-\sum_{k=-\infty}^{\infty} \frac{8 \pi^{2} V_{0} p_{0} k^{2}}{\left((\pi k)^{4}-\lambda\right)^{2}}-\sum_{\substack{m, k=-\infty \\
m \neq k}}^{\infty} F(k, m, \lambda), \\
& F(k, m, \lambda)=\frac{\pi^{2}\left(k^{2}+m^{2}\right) \widehat{V}_{m-k} \widehat{p}_{m-k}}{\left((\pi m)^{4}-\lambda\right)\left((\pi k)^{4}-\lambda\right)},
\end{aligned}
$$

where the series converge uniformly on each contour $K_{n}, n \in \mathbb{N}$. Moreover, the identity $\oint_{K_{n}} \frac{d \lambda}{\left((\pi k)^{4}-\lambda\right)^{2}}=0$ and the decomposition $\{|k| \neq|m|\}=D_{1} \cup D_{2} \cup D_{3}$, where $D_{1}, D_{2}, D_{3}$ are given by

$$
\begin{array}{r}
D_{1}=\{|k| \neq|m|,|k| \leqslant n,|m| \leqslant n\} \cup\{|k| \neq|m|,|k|>n,|m|>n\}, \\
D_{2}=\{|k| \leqslant n,|m|>n\}, \quad D_{3}=\{|k|>n,|m| \leqslant n\},
\end{array}
$$

give

$$
\begin{aligned}
& \oint_{K_{n}} \operatorname{Tr}\left(V \mathcal{R}_{2}^{0}\left(h_{2}^{0} p+p h_{2}^{0}\right) \mathcal{R}_{2}^{0}\right) d \lambda \\
& =\oint_{K_{n}} \sum_{\substack{m, k=-\infty \\
m \neq k}}^{\infty} F(k, m, \lambda) d \lambda=I_{1}(\lambda)+I_{2}(\lambda)+I_{3}(\lambda)
\end{aligned}
$$

for all $n \in \mathbb{N}$, where

$$
I_{j}(\lambda)=\oint_{K_{n}} \sum_{D_{j}} F(k, m, \lambda) d \lambda .
$$

We have $I_{1}(\lambda)=0$ and thus we need to consider $I_{2}, I_{3}$.

Consider $I_{3}$, the proof for $I_{2}$ is similar. Identity (3.11) gives

$$
I_{3}(\lambda)=\oint_{K_{n}} \sum_{D_{3}} F(k, m, \lambda) d \lambda=\frac{2}{i \pi} \sum_{D_{3}} \frac{\widehat{V}_{m-k} \widehat{p}_{m-k}}{m^{2}-k^{2}} .
$$


Consider the case $k>n,|m| \leqslant n$, the proof for the other case is similar. Using $V \in W_{2}^{1}(0,2)$ and $p \in W_{4}^{1}(0,2)$ we obtain

$$
\frac{\left|\widehat{V}_{m-k} \widehat{p}_{m-k}\right|}{\left|m^{2}-k^{2}\right|} \leqslant \frac{C}{|k-m|^{6}\left|m^{2}-k^{2}\right|} \leqslant \frac{C}{|k-m|^{7}(|m|+|k|)}
$$

for some constant $C$. Define $k^{\prime}=k-n \geqslant 1$ and $m^{\prime}=n-m \in[0,2 n]$. Then we obtain

This yields

$$
\frac{1}{|k-m|^{7}(|m|+|k|)} \leqslant \frac{1}{\left|k^{\prime}+m^{\prime}\right|^{7} n} \text {. }
$$

$$
\sum_{k>n,|m| \leqslant n} \frac{\left|\widehat{V}_{m-k} \widehat{p}_{m-k}\right|}{\left|m^{2}-k^{2}\right|} \leqslant \sum_{k^{\prime} \geqslant 1, m^{\prime} \geqslant 0} \frac{C}{\left|k^{\prime}+m^{\prime}\right|^{7} n}=\frac{C_{1}}{n}, \quad C_{1}=\sum_{k^{\prime} \geqslant 1, m^{\prime} \geqslant 0} \frac{C}{\left|k^{\prime}+m^{\prime}\right|^{7}} .
$$

Similar arguments show that

$$
\sum_{k<-n,|m| \leqslant n} \frac{\left|\widehat{V}_{m-k} \widehat{p}_{m-k}\right|}{\left|m^{2}-k^{2}\right|} \leqslant \frac{C_{2}}{n}
$$

for some $C_{2}>0$ and then (3.12) yields $I_{3}(\lambda)=O\left(n^{-1}\right)$ as $n \rightarrow \infty$. Similar estimates yield $I_{2}(\lambda)=O\left(n^{-1}\right)$. Then (3.11) gives

$$
\oint_{K_{n}} \operatorname{Tr}\left(V \mathcal{R}_{2}^{0}\left(h_{2}^{0} p+p h_{2}^{0}\right) \mathcal{R}_{2}^{0}\right) d \lambda=O\left(n^{-1}\right)
$$

Substituting this asymptotics and (3.9) into (3.8) we obtain (2.13).

Acknowledgments. Various parts of this paper were written during Evgeny Korotyaev's stay in Mittag-Leffler Institute, Sweden and Centre for Quantum Geometry of Moduli spaces (QGM), Aarhus University, Denmark. He is grateful to the institutes for the hospitality. His study was supported by the Ministry of education and science of Russian Federation, project 07.09.2012 No 8501 and the RFFI grant "Spectral and asymptotic methods for studying of the differential operators" No 11-01-00458 and partly supported by the Danish National Research Foundation grant DNRF95 (Centre for Quantum Geometry of Moduli Spaces - QGM). Andrey Badanin's study was supported by the Ministry of education and science of Russian Federation, project No 1.5711.2011.

\section{References}

[1] Badanin, A., Korotyaev, E. Sharp spectral asymptotics for fourth order operators on the circle. ArXiv:1309.3447 (2013).

[2] Badanin, A., Korotyaev, E. Sharp spectral asymptotics for fourth order operators on the unit interval. ArXiv:1309.3449 (2013).

[3] Badanin, A., Korotyaev, E. Spectral estimates for periodic fourth order operators, St.Petersburg Math. J. 22:5 (2011) 703-736.

[4] Badanin, A., Korotyaev, E. Even order periodic operators on the real line. International Mathematics Research Notices, 2012(5) (2012), 1143-1194.

[5] Caudill Jr, L. F., Perry, P. A., Schueller, A. W. Isospectral sets for fourth-order ordinary differential operators. SIAM journal on mathematical analysis, 29(4) (1998), 935-966.

[6] Dubrovin, B. Periodic problems for the Korteweg - de Vries equation in the class of finite band potentials Functional Analysis and Its Applications, 9:3 (1975), 215-223.

[7] Its, A. R., Matveev, V. B. Schrodinger operators with finite-gap spectrum and N-soliton solutions of the Korteweg-de Vries equation. Teoreticheskaya i Matematicheskaya Fizika, 23(1) (1975), 51-68. 
[8] Korotyaev, E. Inverse Problem and the trace formula for the Hill Operator, II. Mathematische Zeitschrift, 231(2) (1999), 345-368.

[9] Levitan, B. M., Sargsyan, I. S. Sturm-Liouville and Dirac Operators. Kluwer, Dordrecht, 1991.

[10] McKean, H. P. Boussinesq's equation on the circle. Communications on Pure and Applied Mathematics, 34(5) (1981), 599-691.

[11] McKean, H. P., van Moerbeke, P. The spectrum of Hill's equation. Inventiones Mathematicae, 30(3) (1975), 217-274.

[12] McLaughlin, J. R. An inverse eigenvalue problem of order four-an infinite case. SIAM Journal on Mathematical Analysis, 9(3) (1978), 395-413.

[13] Magnus, W., Winkler, S. Hill's equation. Courier Dover Publications, 1979.

[14] Mikhailets, V. A., Molyboga, V. M. On the spectrum of singular perturbations of operators on the circle. Mathematical Notes, 91(4) (2012), 588-591.

[15] Nazarov, A.I., Stolyarov, D.M., Zatitskiy, P.B. Tamarkin equiconvergence theorem and trace formula revisited, ArXiv:1210.8097 (2012).

[16] Ostensson, J. Trace Formulae for Fourth Order Differential Operators and their Applications. Diss. KTH, 2004.

[17] Papanicolaou, V. The spectral theory of the vibrating periodic beam. Comm. Math. Phys. 170 (1995), no. 2, 359-373.

[18] Papanicolaou, V. The Periodic Euler-Bernoulli Equation, Transactions of the American Mathematical Society 355, No. 9(2003), 3727-3759.

[19] Papanicolaou, V. G., Kravvaritis, D. An inverse spectral problem for the Euler-Bernoulli equation for the vibrating beam. Inverse Problems, 13(4) (1997), 1083.

[20] Sadovnichii, V. A. The trace of ordinary differential operators of high order. Matematicheskii Sbornik, 114(2) (1967), 293-317.

[21] Sadovnichii, V. A. Trace formulae for ordinary differential operators of higher orders. Matematicheskie Zametki, 1(2), (1967), 179-188.

[22] Sadovnichii, V. A., Podol'skii, V. E. Traces of operators. Russian Mathematical Surveys, 61(5) (2006), 885-953.

[23] Trubowitz, E. The inverse problem for periodic potentials. Communications on Pure and Applied Mathematics, 30(3) (1977), 321-337.

[24] Tkachenko, V. Eigenfunction expansions associated with one-dimensional periodic differential operators of order 2n. Functional Analysis and Its Applications, 41(1) (2007), 54-72.

Northern (Arctic) Federal University, Northern Dvina emb, 17, Arkhangelsk, 163002, RUSSIA

Mathematical Physics Department, Faculty of Physics, Ulianovskaya 2, St. PetersBurg State University, St. Petersburg, 198904, Russia 